\title{
Wie hätten Sie entschieden?
}

\author{
Oswald Hasselmann ${ }^{a}$, Thomas Schmitt ${ }^{\mathrm{b}}$, Bernard Baertschi ${ }^{\mathrm{c}}$ \\ ${ }^{a}$ Leitender Arzt Neuropädiatrie - Klinische Ethik, Ostschweizer Kinderspital, St. Gallen; ${ }^{b}$ Leitender Arzt Neuropädiatrie, Co-Chefarzt, Kantonsspital Luzern; \\ 'Institute for Biomedical Ethics, University Medical School, Geneva
}

Im November 2018 wurde im Rahmen der Jahrestagung der Gesellschaft für Neuropädiatrie in Genf ein Sitzungsblock dem Thema «ethischer Umgang mit neu zur Verfügung stehenden Therapien» gewidmet (Abb. 1). Geleitet wurde diese Sitzung von zwei Neuropädiatern aus St. Gallen und Luzern sowie einem Ethiker aus Genf. Ausgangspunkt war das zunehmende Unbehagen innerhalb der neuropädiatrischen Ärzteschaft über die immensen Preisanstiege von neu etablierten

\section{Ausgangspunkt war das zunehmende Unbehagen über die immensen Preisanstiege von neu etablierten Therapieverfahren.}

Therapieverfahren für seltene Erkrankungen verbunden mit der Sorge, dass die Rechnungsbegleichung neuerer Therapieverfahren zu Lasten etablierter Therapien gehen könnte. Die Teilnehmer der Tagung wurden zu Beginn und nach einem zusammenfassenden Überblick über den gesundheitspolitischen Rahmen der Diskussion gebeten, sich anonym zu äussern, wie sie sich zu spezifischen Fragen der Kosten-Effektivität am Beispiel von zwei neuen Therapieverfahren

\section{Résumé}

En novembre 2018, lors du congrès annuel des neuropédiatres suisses à Genève, un bloc de séances a été consacré au thème "utilisation éthique de nouveaux traitements disponibles» (fig. 1). Cette séance était présidée par deux neuropédiatres de Saint-Gall et de Lucerne et un éthicien de Genève. Le point de départ était le malaise croissant au sein du corps médical des neuropédiatres face à l'envolée considérable des prix des nouvelles procédures thérapeutiques pour les maladies rares, associé à la crainte que le règlement de leurs factures ne se fasse au détriment de celui des traitements établis. Les participants à la réunion ont été priés, au début et après une vue d'ensemble récapitulative du cadre de la politique de santé du débat, d'exprimer anonymement leurs positions sur des questions spécifiques du rapport coût-efficacité, en se basant sur l'exemple de deux nouvelles méthodes thérapeutiques. Comme il s'agit là de questions auxquelles les médecins de toutes les disciplines peuvent être confrontés, nous estimons que la publication dans le Bulletin des médecins suisses est utile pour proposer aux lecteurs des pistes de positionnement par rapport à leur spécialité.

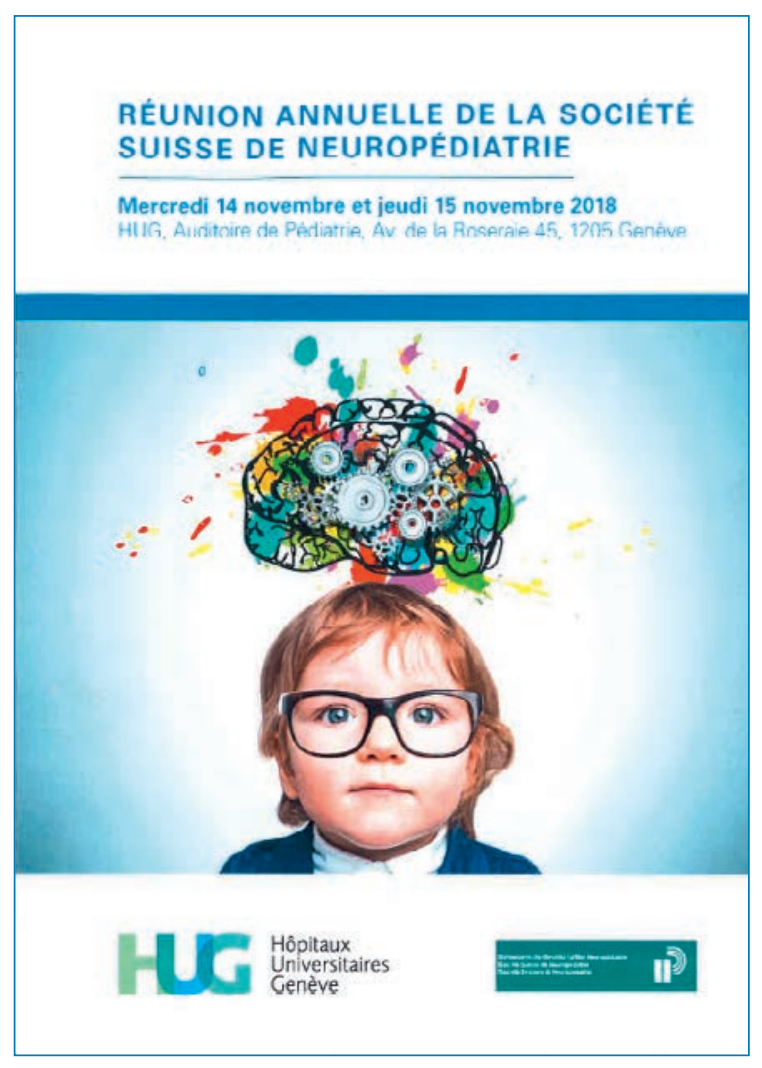

Abbildung 1: Deckblatt des Tagungsflyers.

positionieren würden. Da es sich hierbei um Fragen handelt, mit denen Ärzte sämtlicher Fachrichtungen konfrontiert werden können, scheint die Veröffentlichung in der Schweizerischen Ärztezeitung sinnvoll, um den Lesern Anregungen für ihre jeweils fachbezogene Positionierung zu geben.

\section{Orphan Drugs für seltene Erkrankungen}

Seltene Erkrankungen werden primär über ihre Prävalenz definiert (Grenzwert in Europa: <1:2000). Aktuell wird von ca. 8000 seltenen Erkrankungen ausgegangen, zu denen pro Jahr ca. 200 meist genetisch definierte neue Krankheitsidentitäten hinzukommen, von denen ca. 10\% aufgrund der prekären Versorgungssituation als "orphan disease» anerkannt werden. Die Hälfte dieser Erkrankungen manifestiert sich bereits in der Kindheit. Sie sind meist durch einen chronischdegenerativen Krankheitsverlauf mit einer Lebens- 
erwartung von weniger als fünf Jahren bei 30\% der Betroffenen gekennzeichnet. Eine nachhaltig wirksame Therapieoption (z.B. mit Enzymersatz-, Antikörper- oder einer genetisch wirksamen Therapie) existiert bei lediglich 1\% der Erkrankten. Es wird davon ausgegangen, dass eine Pädiaterin in ihrer Praxis nicht mehr als ein Kind pro Jahr mit einer behandelbaren seltenen Erkrankung sieht [1].

\section{Das Nationale Konzept}

Aus dem Bestreben, die gesundheitliche Situation von Menschen mit seltenen Erkrankungen zu verbessern, wurde im Oktober 2014 in der Schweiz das Nationale Konzept Seltene Erkrankungen etabliert und ein 19-teiliger Massnahmenkatalog formuliert, der sich an vergleichbaren Aktivitäten in den europäischen Nachbarländern orientiert. Der Zielkatalog des Konzeptes fokussiert auf folgende Aufgabenschwerpunkte: Unterstützung der Patientinnen, ihrer medizinischen Betreuer und der verwaltenden Dienste bei der Sicherstellung des Zuganges zu einer Diagnose, der Therapie und der Vergütung verbunden mit einer fortlaufenden nationalen und international vernetzten Forschung auf diesem Gebiet. Zur spezifischen Umsetzung wurde 2017 die Nationale Koordination Seltene Krankheiten (kosek) etabliert. Diese ist aktuell in die Anerkennung von Kompetenznetzwerken und Referenzzentren involviert, welche die Vernetzung, die Aus-, Weiter- und Fortbildung, die Erstellung von Guidelines sowie das Forschungs- und Datenmanagement für prädefinierte Erkrankungen sicherstellen soll [2].

\section{Unterstützung bei den Zulassungs- verfahren}

Ursprünglich war man davon ausgegangen, dass es wenig attraktiv sei, Arzneimittel für solche Erkrankungen zu entwickeln, da eine Amortisierung der Forschungs- und Entwicklungsausgaben kaum erreicht werden könne. Es wurden daher in den Pharmaka produzierenden Ländern finanzielle und organisatorische Anreize (u.a. eine 10- bis 12-jährige Marktexklusivität und Gebührenbefreiungen) für die Erforschung, die Entwicklung und das Inverkehrbringen von Arzneimitteln für seltene Leiden geschaffen. Seitdem 1983 der Orphan Drug Act in den USA wirksam wurde, lässt sich in der Folge hier wie in Europa und Asien eine stetige Verlagerung der Neuzulassung von Medikamenten in einen nunmehr profitablen Orphan-Drug-Bereich beobachten [3]. Parallel hierzu beobachten wir auch in der Schweiz eine stetige Zunahme des Anteils am Bruttosozialprodukt, der durch krankheitsassoziierte
Kosten verursacht wird (1960: 6\%; 2016: 12\%; Tendenz weiter steigend). Vor diesem Hintergrund werden aktuell in der Schweiz verschiedene Rationierungsmassnahmen (siehe u.a. die Auseinandersetzungen um das sogenannte "Globalbudget») diskutiert [4].

Anhand von zwei neu zugelassenen Orphan Drugs soll beispielhaft gezeigt werden, wie die Ermöglichung von neuen therapeutischen Optionen mit gesundheitspolitischen Herausforderungen einhergeht.

\section{Nursinersen}

Ein kürzlich gegen die spinale Muskelatrophie zugelassenes Medikament, welches mit Unterstützung der Patientenorganisation (SMA Foundation, Cure SMA) entwickelt wurde und seine Wirkung über einen alternativen Spleissvorgang auf mRNA-Ebene entwickelt, wird den Kostenträgern mit Kosten im ersten Jahr von 600000 CHF in Rechnung gestellt. Erste Erfahrungen mit dem Medikament deuten darauf hin, dass die Therapiewirkung mit zunehmendem zeitlichem Abstand zur Erstmanifestation nachlässt. Unter Berücksichtigung der Kosteneffektivität bei Berechnung der durch die Therapie gewonnenen zusätzlichen Lebensqualität stellt sich die Frage, ob der hohe Preis für alle Unterformen der Erkrankung (Typ 1-3) zu erstatten ist. In einigen Staaten der USA und in $\mathrm{Ka}$ nada wurde im Gegensatz zur Schweiz die Zulassung auf die Untergruppe I der SMA beschränkt. In Grossbritannien wurde das Medikament aufgrund von qualitätskorrigierten Lebensjahren (englisch; qualityadjusted life year oder QUALY) mit einem geforderten finanziellen Einsatz weit oberhalb der prädeterminierten Schwelle von 100000 GBP pro gewonnenes Qualitätsjahr bislang nicht zur Kostenerstattung innerhalb des NHS zugelassen. Die Invalidenversicherung hat die fortgesetzte Kostenerstattung für alle Subtypen der SMA über das erste Therapiejahr hinaus zugesichert, insofern ein definierter Therapieerfolg dokumentiert werden kann [5].

\section{Everolimus}

Ein immunsuppressiver, anti-proliferativer Wirkstoff (mTOR-Inhibitor) wurde zugelassen für Patienten mit einer tuberösen Sklerose, welche an einem nicht operablen, gutartigen subependymalen Riesenzellastrozytom leiden. Die Zulassung wurde 2017 erweitert auf die Behandlung von einer mit der tuberösen Sklerose assoziierten fokalen Epilepsie, sofern diese nicht auf gängige Antikonvulsiva anspricht. Es wird davon ausgegangen, dass diese Therapie, zu Jahreskosten von $60000 \mathrm{CHF}$, lebenslang eingenommen werden muss, sofern der immunsuppressive Effekt nicht einen The- 
rapiestopp erzwingt. Da bislang keine Daten über Langzeiteffekte im Kindesalter vorliegen und die Therapiekosten ca. 20-mal höher als bei einer antikonvulsiven Therapie liegen, wird zu einer vorausschauenden Nutzen-Risiko-Evaluation geraten [6].

\section{Ein anspruchsvoller Interessenausgleich}

Das Bundesamt für Gesundheit (BAG) steht vor dem Hintergrund der beschriebenen politischen Vorgaben und der aktuellen Therapieentwicklungen mit hochpreisigen Medikamenten vor der Aufgabe, die Sicherung der medizinischen Grundversorgung zu gewährleisten und einen Konsens für notwendige Rationierungsmassnahmen zu finden. Während die Zulassung eines Medikamentes in der Schweiz bislang an die WZW-Kriterien (wirksam, zweckmässig, wirtschaftlich) geknüpft war, werden bei den Orphan Drugs deutlich weniger stringente Anforderungen an den Wirksamkeitsnachweis gestellt. Das Primat der Wirtschaftlichkeit ist bei Jahrestherapiekosten von bis zu einer halben Million Franken nicht mehr uneingeschränkt einsichtig.

\section{Medizinethische Betrachtung}

Für die betreuenden Ärzte bzw. ihre Standesorganisationen ergibt sich zunehmend ein ethisches Dilemma zwischen dem Primat der Verpflichtung für den individuellen Patienten und jener für die gesellschaftlich basierte Solidargemeinschaft. Philosophisch spricht man von einer Spannung zwischen einem deontologischen und einem utilitaristischen Ansatz. Die Entscheidungsträger können die divergierenden Interessenlagen dem freien Spiel der Marktkräfte überlassen oder sich bemühen, dem Anspruch eines Gerechtigkeitsansatzes zu folgen. Einen konzeptionellen Rahmen kann die "Maximin-Regel» des Philosophen John Rawls geben, welche zum Ziel hat, einerseits für alle ein ausreichendes minimales Versorgungsniveau sicherzustellen und dort, wo sich eine Rationierung nicht vermeiden lässt, diese so zu gestalten, dass die am schlechtesten Gestellten den geringsten Schaden erleiden [7].

\section{Änderungen der Rahmenbedingungen}

Die European Medicines Agency (EMA) hat sich dieser Vorgabe gestellt, indem sie einen "adaptive pathways approach" für die weitere Finanzierung der Orphan Drugs entwickelt hat, der die fortgesetzte Finanzierung u.a. abhängig macht von Effektivitäts- vorgaben durch transparent durchgeführte Langzeitstudien. Andere diskutierte Lösungsansätze sind eine engere Definition von Orphan Drugs, eine verkürzte Monopolstellung, eine stärkere Mitsprache bei der Preisfestsetzung durch sämtliche Stakeholder und eine engere Bindung des Verkaufspreises an die dokumentierte Langzeiteffektivität und an die nachvollziehbaren Entwicklungskosten der Hersteller [8]. Aus der Überzeugung, dass sich die für die Therapie verantwortlichen Ärzte parallel zu den Zulassungsbehörden, den Patientenorganisationen und den Herstellern aktiv in den Entscheidungsprozess über die Finanzierungsmöglichkeiten innerhalb des Grundversicherungssystems einbringen können, wurden die Tagungsteilnehmer zu Anfang ( $\mathrm{n}=76)$ und zum Ende $(\mathrm{n}=$ 81) unserer hier zusammengefassten Darstellung um Antworten auf die folgenden Positionen bezüglich des prioritären Umgangs mit neuen und effektiven Medikamenten gebeten; jeweils zwei Optionen waren möglich

1. Kostenerstattung ohne Einschränkung

2. Versicherungsbeiträge in Abhängigkeit von der Dringlichkeit der Medikation

3. Zugang zu den Medikamenten nur bei Ansprechen der Therapie

4. Koppelung des Verkaufspreises an die Effektivität

5. Keine Kostenerstattung, falls die QALY-Schwelle von 100000 CHF überschritten wird

6. Keine Kostenerstattung für hochpreisige Medikamente durch die Grundversicherung

7. Kostenerstattung nur, wenn die Patente an die Öffentlichkeit zurückgegeben werden

\section{Auswertung}

Während vor den Ausführungen knapp 15\% für eine uneingeschränkte Kostenerstattung votierten, wurde diese Position bei der zweiten Befragung nur noch von $5 \%$ vertreten. Die meisten Teilnehmer wären einverstanden mit einer Koppelung der Kostenerstattung an den Nachweis einer Effektivität. Nach dem Vortrag stieg diese Einschätzung von $28 \%$ auf $43 \%$. Das im angelsächsischen Raum schon etablierte QALY-System wurde unabhängig von den Ausführungen nur von weniger als $10 \%$ der Befragten als ein sinnvolles Instrument für einen Interessenausgleich angesehen. Ein Unbehagen über die begrenzte öffentliche Einflussnahme bei der Preisgestaltung zeigte sich bei ca. einem Fünftel der Teilnehmer bei der Frage, ob Patente für hochpreisige Medikamente ggf. von privater in die öffentliche Hand überführt werden sollen, um dadurch die Versorgung für alle Bedürftigen sicherzustellen (Abb. 2). 


\section{Die Antworten der befragten Neuropädiater}

\section{What are your $\mathbf{2}$ top-priorities? Are your $\mathbf{2}$ top-priorities the same? Very expensive and "effective" new drug \\ Very expensive and "effective" new drug}

1. Reimbursement without restriction $14.5 \%$

2. Insurance contribution according to needs

$15.8 \%$

3. Limit access according to treatment response $27.6 \%$

4. Floating saleprices dependent on efficacy $5.3 \%$

5. Deny access if above QALY-limit (e.g. Fr. 100000)

$9.2 \%$

6. Deny access within the public insurancesystem | $1.3 \%$

1. Reimbursement without restriction $4.9 \%$

2. Insurance contribution according to needs $19.8 \%$

3. Limit access according to treatment response

4. Floating saleprices dependent on efficacy

4. $3.7 \%$

5. Deny access if above QALY-limit (e.g. Fr. 100000)

$7.4 \%$

6. Deny access within the public insurancesystem $2.5 \%$

7. Reimbursement only if patents remain public $26.3 \%$

7. Reimbursement only if patents remain public $18.5 \%$

Abbildung 2: Meinungsbild der befragten Neuropädiater im November 2018.

\section{Résumé und Ausblick}

Als betreuende Ärzte haben wir eine Verantwortung sowohl für unsere Patienten als auch für das Gesundheitssystem, in dem wir arbeiten. Aus dem gut nachvollziehbaren Bemühen, Menschen mit seltenen Erkrankungen ein besseres Versorgungsangebot zu ermöglichen, hat sich durch die zunehmende Zulassung von sehr teuren Medikamenten zu Lasten der Grundversicherung ein finanzielles Ungleichgewicht entwickelt, das den Fortbestand des solidarisch finanzierten Gesundheitssystems in der Schweiz gefährdet. Das Unbehagen über diese Entwicklung wurde von den Veranstaltern des Jahreskongresses der Schweizer Gesellschaft für Neuropädiatrie aufgenommen und führte zu einer interaktiv gestalteten Sitzung mit klinischen und medizinethischen Aspekten, zu denen sich die Teilnehmer zweimal aktiv einbringen konnten. Auch wenn die Voten nicht den Anspruch erheben können, die Meinung der Schweizer Neuropädiater insgesamt wiederzugeben, so deuten sie doch auf die Notwendigkeit hin, das Nationale Konzept Seltene Erkrankungen um den Auftrag einer gerechten und nachhaltigen Kostenverteilung zwischen Produzenten, Erkrankten und der Allgemeinheit zu ergänzen. Hierfür gibt es auf nationaler und auf internationaler Ebene unterschiedliche Vorschläge, um deren Prüfung wir uns als Ärzte bemühen sollten.

\section{Disclosure statement}

Interessenverbindungen bestehen bei den Initiatoren der Sitzung und dem Artikelautor nicht.

Literatur

1 Lazor R, Sizonenko LD. Seltene Erkrankungen: 30 Millionen Menschen in Europa sind betroffen. Schweiz Ärzteztg. 2008.

2 https://www.kosekschweiz.ch/grundlagen/nationales-konzept (30.11.2018).

3 Kwon D. How Orphan Drugs Became a Highly Profitable Industry. The Scientist. 2018.

4 Positionspapier der FMH, Gesundheitswesen Schweiz, 2016.

5 Gidarot T, Servais L. Nusinersen treatment of spinal muscular atrophy: current knowledge and existing gaps. Developmental Medicine \& Child Neurology. 2018.

6 Curatolo P, Franz DN, Lawson JA, Yapici Z, Ikeda H, Polster T. Adjunctive everolimus for children and adolescents with treatment-refractory seizures associated with tuberous sclerosis complex. Lancet Child Adolesc Health. 2018.

7 Rawls J. Eine Theorie der Gerechtigkeit. 1971.

8 https://www.ema.europa.eu/en/human-regulatory/researchdevelopment/adaptive-pathways (30.11.2018). 